\title{
WISP1 mediates lung injury following hepatic ischemia reperfusion dependent on TLR4 in mice
}

\author{
Yao Tong ${ }^{\dagger}$, Zhuang Yu ${ }^{\dagger}$, Renlingzi Zhang ${ }^{\dagger}$, Xibing Ding, Zhixia Chen and Quan Li
}

\begin{abstract}
Background: Hepatic ischemia-reperfusion injury (IRI) is a common pathological phenomenon, which causes hepatic injury as well as remote organ injuries such as the lung. Several mediators, such as oxidative stress, $\mathrm{Ca}^{2+}$ overload and neutrophil infiltration, have been implied in the pathogenesis of liver and remote organ injuries following reperfusion. WNT1 inducible signaling pathway protein 1 (WISP1) is an extracellular matrix protein that has been associated with the onset of several malignant diseases. Previous work in our group has demonstrated WISP1 is upregulated and contributes to proinflammatory cascades in hepatic IRI. However, the role of WISP1 in the pathogenesis of lung injury after hepatic IRI still remains unknown.
\end{abstract}

Methods: Male C57BL/6 mice were used to examine the expression and role of WISP1 in the pathogenesis of lung injuries after hepatic IRI and explore its potential mechanisms in mediating lung injuries.

Results: We found WISP1 was upregulated in lung tissues following hepatic IRI. Treatment with anti-WISP1 antibody ameliorated lung injuries with alteration of cytokine profiles. Administration with rWISP1 aggravated lung injuries following hepatic IRI through excessive production of proinflammatory cytokines and inhibition of anti-inflammatory cytokines.

Conclusions: In this study, we concluded that WISP1 contributed to lung injuries following hepatic IRI through TLR4 pathway.

Keywords: Hepatic ischemia-reperfusion injury, WNT1 inducible signaling pathway protein 1, Inflammatory cascades, Toll like receptor

\section{Introduction}

Hepatic ischemia-reperfusion injury (IRI) is a pathological phenomenon event when hypoxic liver undergoes oxygenic blood reperfusion. Usually IRI can be divided into two categories, namely warm ischemia which often occurs in trauma, shock, and liver transplantation with temporary blood interruption and cold ischemia which appears during organ preservation before transplantation. Recent studies have implied several pathological mechanisms in the pathogenesis of IRI including production of reactive oxygen species (ROS), synthesis of inducible nitric oxide synthase (iNOS), and secretion

\footnotetext{
* Correspondence: quanligene@126.com

${ }^{\dagger}$ Yao Tong, Zhuang Yu and Renlingzi Zhang contributed equally to this work. Department of Anesthesiology, Shanghai East Hospital, School of Medicine, Tongji University, 150 Jimo Road, Shanghai 200120, China
}

of proinflammatory cytokines and chemokines which leads to immune cell (especially neutrophil) recruitment and inflammatory cascades [1-3]. Excessive production of proinflammatory cytokines in the serum, including TNF- $\alpha$, IL- $1 \beta$ and IL- 6 in the early phase contribute to the local and remote organ damage $[4,5]$. Previous studies have confirmed lung injury in the IRI model [6]. However, the exact mechanism of IRI induced remote organ injury still remains unclear.

Pattern recognition receptors (PRRs) have been demonstrated to participate in the pathogenesis of IRI, with one of the most important members being Toll-like receptor 4 (TLR4) [7]. TLR4 can recognize various kinds of endogenous antigens and is activated in IRI in liver and remote organs [8]. The functions of TLR4 on immune cells are more vital than on hepatocytes 
although the regulating mechanisms may be different [9, 10]. Besides TLR4 signaling, other PRRs, such as TLR2 and TLR9, have been reported to be involved in the pathogenesis of IRI [11-13].

WNT1 inducible signaling pathway protein 1 (WISP1) is a secreted extracellular matrix (ECM) protein which is ubiquitously expressed in multiple organs, such as lung, liver, kidney, heart and small intestine [14]. WISP1 belongs to the $\mathrm{CCN}$ family which contains 6 members, namely CCN1 (cysteine-rich protein 61, Cyr61), CCN2 (connective tissue growth factor, CTGF), CCN3 (nephroblastoma overexpressed gene, NOV), CCN4 (WNT1 inducible signaling pathway protein-1, WISP1), CCN5 (WISP2) and CCN6 (WISP3) [15]. The functions of WISP1 has been linked to cell proliferation, survival and differentiation [15]. Recent studies have demonstrated that WISP1 relative expression is significantly upregulated in some diseases, including lung carcinoma, hepatocellular carcinoma [16] and colon adenocarcinomas [17]. Interestingly, Li et al. have linked WISP1 to the progression of inflammation [18], that is, stimulation of recombinant WISP1 aggravates proinflammatory responses in LPS stimulated macrophages. Previous work in our group has demonstrated WISP1 is upregulated and contributes to proinflammatory cascades in hepatic IRI [19]. However, the role of WISP1 in the pathogenesis of lung injury after IRI still remains unknown.

In this study, we aimed to investigate the WISP1 expression in the lung tissue after hepatic IRI and determine the regulating mechanisms and functions of WISP1 in the lung injury after hepatic IRI.

\section{Methods}

\section{Mice}

C57BL/6 mice were purchased from Shanghai Laboratory Animal Co Ltd. (SLAC, Shanghai, China). TLR4 knockout (TLR4 KO) mice were kindly provided by Dr. Timothy R. Billiar (University of Pittsburgh, USA). All mice were raised in specific pathogen-free condition. Male mice of $8-10$ weeks (weight 18.4.8 \pm $1.8 \mathrm{~g}$ ) old were used for experiments. Animal experiments were authorized by the Ethics Committee of Tongji University.

\section{Induction of hepatic I/R injury model]}

The induction of segmental (70\%) hepatic hepatic warm $\mathrm{I} / \mathrm{R}$ injury model was performed as previously described with minor modifications [8, 20, 21]. Mice were treated with isotype control IgG or neutralizing WISP1/WISP2 antibody (MyBioSource, $6 \mu \mathrm{g} / \mathrm{g}$ ) intraperitoneally $1 \mathrm{~h}$ before ischemia and again at the time of reperfusion. In another experiment, mice were administered with recombinant WISP1 protein (rWISP1, $1 \mu \mathrm{g} / \mathrm{g}$ ) or sterile phosphate-buffered saline (PBS, w/o $\mathrm{Ca}^{2+} / \mathrm{Mg}^{2+}$ ) intraperitoneally immediately after reperfusion. Sham mice were sufficiently anesthetized, and then a midline abdominal incision was made. The portal triad was exposed without further treatment for liver ischemia. Mice were sacrificed at the predetermined time points $(0 \mathrm{~h}, 3 \mathrm{~h}, 6 \mathrm{~h}, 12 \mathrm{~h}$ and $24 \mathrm{~h})$ after reperfusion for collecting serum and lung tissues.

\section{Lung edema measurement}

Lung edema was evaluated as previously reported by an increase in the wet-to-dry (W/D) weight ratio of the lungs $[21,22]$. The left lung was dissected and weighted before and after drying in a micro oven at $65^{\circ} \mathrm{C}$ for $48 \mathrm{~h}$. $\mathrm{W} / \mathrm{D}$ ratio was then detected.

\section{Alveolar-capillary permeability assay}

Evans blue albumin (EBA) was used to measure Alveolar-capillary permeability as previous illustrated [23]. Briefly, the internal jugular vein of mice was injected with EBA $(25 \mathrm{mg} / \mathrm{kg}) 1 \mathrm{~h}$ before sacrificed. And then, the right lung and blood samples were available for further steps.

\section{Evaluation of Bronchoalveolar lavage fluid (BALF)}

BALF was evaluated as previously publications in our group [21]. Mice were instilled with $1 \mathrm{~mL}$ PBS and approximately $80 \%$ fluid was retrieved. BALF was kept on ice immediately after recovered and was then centrifuged at $1000 \times \mathrm{g}, 4{ }^{\circ} \mathrm{C}$ for $5 \mathrm{~min}$. Supernatants were used for total protein concentration examination and cytokine levels, which can keep at $-80^{\circ} \mathrm{C}$ for long-term preservation. Total cell counts were determined using a hemocytometer.

\section{RNA extraction, reverse transcription PCR, Quantative real-time PCR}

Total RNA was extracted from lung tissues using TRIzol reagent (Sigma-Aldrich) according to established protocols in our group [21, 24]. The total concentration of RNA was measured at $260 \mathrm{~nm}$ with a spectrophotometer (BeckmanCoulter, Brea, CA, USA). First strand complementary DNA (cDNA) synthesis was performed using PrimerScript RT Master Mix (Takara Bio Inc., Shiga, Japan) according to the manufacturer's protocols. Real-time PCR for glyceraldehyde-3-phosphate dehydrogenase (GAPDH), WISP1, IL-6, TNF- $\alpha$, IL-10 was performed using SYBR premix Ex Taq $^{\text {тм }}$ (Takara) with a Step One Plus real-time PCR system (Applied biosystems, USA), under the manufacturer's instructions. GAPDH was used as house-keeping gene to normalize the gene expression. Gene relative expression was calculated using the $2^{-\Delta \Delta} \mathrm{Ct}$ method. All of the primers were synthesized by Sangon Biotech (Shanghai, China). Primers sequence was as follows: mWISP1 
forward 5' - CGCCCGAGGTACGCAATAG, reverse 5' GCAGTTGGGTTGGAAGGACT, mIL-6 forward 5' CTGCAAGAGACTTCCATCCAG, reverse 5' - AGTG GTATAGACAGGTCTGTTGG, mTNF- $\alpha$ forward $5^{\prime}$ CAGGCGGTGCCTATGTCTC, reverse $5^{\prime}$ - CGAT CACCCCGAAGTTCAGTAG, mIL-10 forward 5'CTTACTGACTGGCATGAGGATCA, reverse 5' GCAGCTCTAGGAGCATGTGG.

\section{Enzyme-linked immunosorbent assay (ELISA)}

BALF supernatants were analyzed for mIL-6, mTNF- $\alpha$ and mIL-10 cytokines using a sensitive commercial ELISA kit (R\&D Systems) according to the manufacturer's instructions.

\section{Western blot analysis}

Western blot analysis was performed as previously described in our studies [21, 24]. Lung tissues were dissected and lysed using iced-cold lysis buffer (Beyotime, catalog no. P0013G). Protein concentration was determined via standard BCA assay. After gel electrophoresis, protein was then transferred to a nitrocellulose membrane and blocked using 5\% nonfat milk for 1 h. Membranes were incubated with rabbit anti-mouse WISP1 antibody (1:400, R\&D systems) and $\beta$-actin antibody (1:10000, Proteintech) at $4{ }^{\circ} \mathrm{C}$ overnight, and then incubated with secondary antibody for $1 \mathrm{~h}$ at $37^{\circ} \mathrm{C}$. Odyssey image analysis system (Licor Biosciences) was used to quantify.

\section{Immunofluorescence}

Immunofluorescent staining was performed using standard protocols $[25,26]$. Briefly, 4-6-mm frozen sections from murine lung tissues were incubated with anti-WISP1 antibody (abcom, 1:200) antibody overnight at $4{ }^{\circ} \mathrm{C}$. Sections were then incubated with secondary Alexa Fluor 488-labelled rabbit anti-mouse antibody (CST, 1:200) for $1 \mathrm{~h}$ at room temperature. Nucleus was stained with DAPI for $5 \mathrm{~min}$ at room temperature. The positive signals were analysed using a confocal fluorescence microscope (Zeiss LSM510 Confocal).

\section{Determination of myeloperoxidase (MPO) level}

The lung MPO level was determined using a commercial mouse MPO ELISA kit (Hycult Biotech) according to the manufacturer's instructions.

\section{Statistical analysis}

Data were displayed as mean \pm SEM. Statistical analysis was performed using GraphPad Prism 5 program. Differences between groups were compared using the Student $t$-test or one-way analysis of variance (ANOVA). Statistically significant differences were considered as $P<0.05$.

\section{Results}

WISP1 is highly increased in lung tissue following hepatic IRI

Previous work in our group has demonstrated WISP1 facilitates proinflammatory cascades following hepatic IRI in the liver dependent on TLR4 signaling [24]. Since remote organ injuries, such as lung injury [8], have been reported following hepatic IRI, we next sought to detect whether WISP1 was involved in the pathogenesis of lung injury in hepatic IRI. Interestingly, WISP1 gene transcription was found to be highly increased in the lung tissue following hepatic IRI after reperfusion for $6 \mathrm{~h}, 12$ h, and $24 \mathrm{~h}$ (Fig. 1a). Western blot analysis further confirmed WISP1 protein level was also significantly upregulated (Fig. 1b). To further analyze WISP1 expression in morphology, we also performed immunofluorescence and Immunohistochemistry assays to verify WISP1 expression. Consistent with previous results, WISP1 is a secreted extracellular matrix protein mainly expressed in cytoplasm (Fig. 1c and d). WISP1 was highly expressed in cytoplasm of the cells in lung tissue (Fig. 1c and d). Together, these results indicated that WISP1 was highly increased in lung tissue following hepatic IRI.

\section{Treatment with anti-WISP1 antibody ameliorates lung injury following hepatic IRI}

To further confirm the role of WISP1 in the pathogenesis of lung injury after hepatic IRI, we treated mice in sham and hepatic IRI group with neutralizing anti-WISP1 or control IgG antibody intraperitoneally $1 \mathrm{~h}$ before ischemia and again at the onset of reperfusion. After reperfusion for $6 \mathrm{~h}$, mice were sacrificed and lung injury was assessed. Histopathology evaluation indicated treatment with anti-WISP1 antibody ameliorated liver injury, as showed by histopathological and biochemical (ALT/AST) evidence (Fig. 2a and $b$ ) and lung injury characterized by less diffuse interstitial edema and inflammatory cell infiltration (Fig. 2c), decreased W/D weight ratio (Fig. 2d) as well as decreased lung EBA permeability (Fig. 2e). Consistent with histopathology analysis, total cell counts (Fig. 2f) and total protein levels (Fig. 2g) were also decreased, indicating less severe inflammation compared with control group. MPO is a peroxidase enzyme which is abundantly expressed on polymorphonuclear neutrophils. The function of MPO has been associated with production of hypochlorous acid $(\mathrm{HOCl})$ which is a subtype of ROS [27]. Consistently, we found MPO levels were also decreased in the lung tissue after anti-WISP1 antibody treatment following hepatic IRI (Fig. 2h). These results indicated that anti-WISP1 neutralizing antibody markedly ameliorated lung injury following hepatic IRI, further confirming the pathological role of WISP1. 

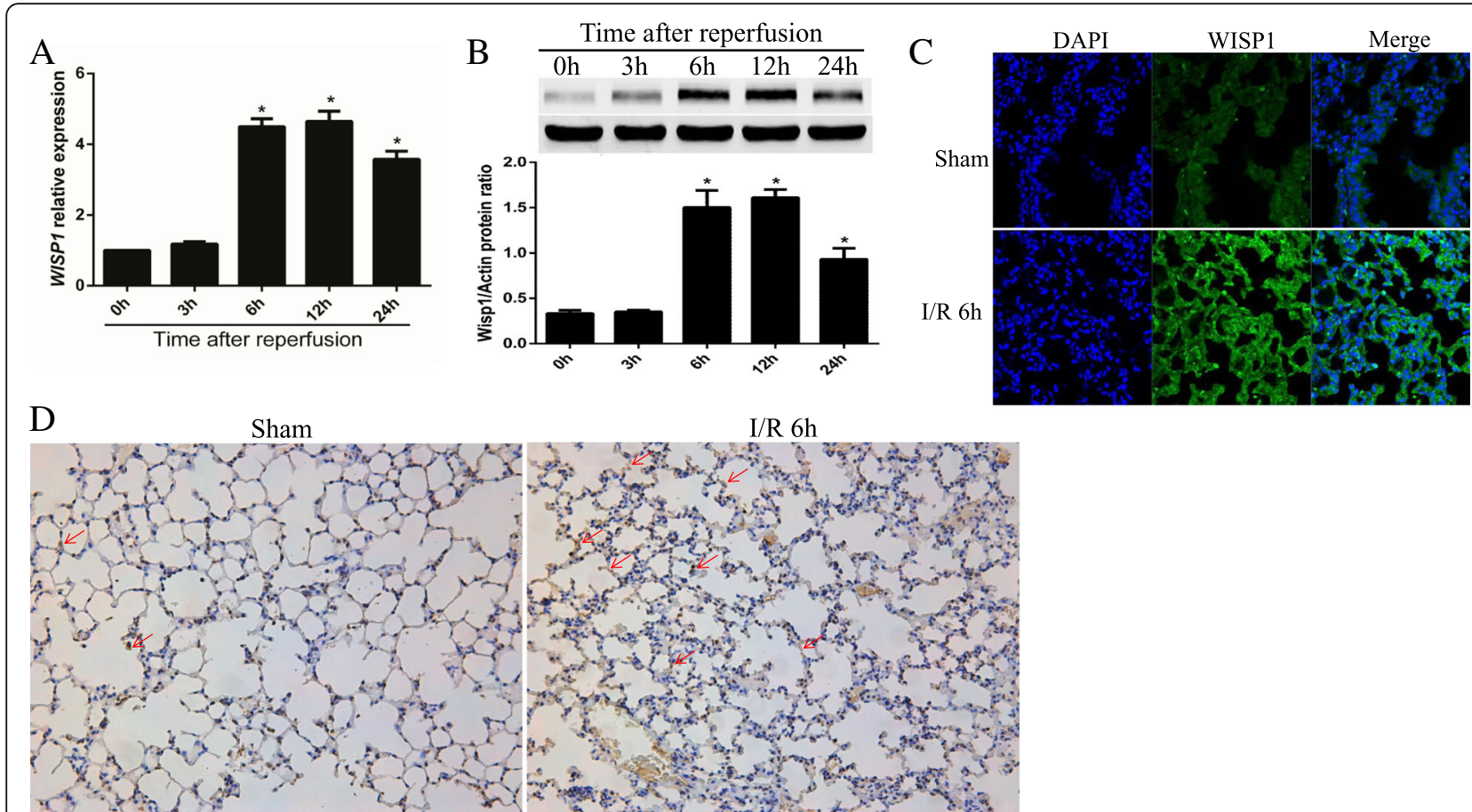

Fig. 1 WISP1 is Highly Increased in Lung Tissue Following Hepatic IRI. QRT-PCR (a) and western blot (b) analysis of WISP1 in lung samples from WT mice undergoing ischemia for 60 min and reperfusion (I/R) for $0 \mathrm{~h}, 6 \mathrm{~h}, 12 \mathrm{~h}$, and $24 \mathrm{~h}$ ( $\mathrm{h}=6$ respectively). c Immunofluorescence and (d) Immunohistochemistry assays of WISP1 in lung samples from WT mice in sham and IRI group undergoing ischemia for 60 min and reperfusion for $6 \mathrm{~h}$. Gene expression was normalized to $\beta$-actin mRNA levels in each sample. ${ }^{*} p<0.05$ compared with $0 \mathrm{~h}$ of reperfusion, data are expressed as mean \pm s.e.m. for all samples

\section{Treatment with anti-WISP1 antibody alters cytokine profiles in lung tissue following hepatic IRI}

Previous results indicated anti-WISP1 antibody ameliorated lung injury following hepatic IRI. We next sought in determine whether treatment with anti-WISP1 antibody augmented proinflammatory or anti-inflammatory cytokine profiles which contributed to the injury in the lung tissue. Mice were treated as previously described. qRT-PCR analysis demonstrated that proinflammatory cytokine IL-6 (Fig. 3a) and TNF- $\alpha$ (Fig. 3b) transcriptions were significantly downregulated in lung tissue following hepatic IRI. However, anti-inflammatory cytokine IL-10 (Fig. 3c) was markedly upregulated. To further determine such cytokine alterations in protein level, we next performed ELISA analysis for these cytokines. In accordance with qRT-PCR results, IL-6 (Fig. 3e) and TNF- $\alpha$ (Fig. 3f) protein level were highly elevated and IL-10 (Fig. 3g) level was decreased. Additionally, we used anti-WISP2 antibody as a control to detect whether it has the same effect. There were no significant changes in pro-inflammatory cytokines' transcription or release following neutralizing WISP2 antibody treatment during liver IRI (Fig. 3d and h). Together, these data suggested anti-WISP1 antibody led to decreased proinflammatory cytokine production and excessive anti-inflammatory cytokine secretion.

\section{Administration with rWISP1 aggravates lung injury following hepatic IRI}

To comprehensively study the pathological role of WISP1, mice in the sham and hepatic IRI group were administered with rWISP1 or control medium (sterile PBS) intraperitoneally once immediately after reperfusion. Lung injury was assessed as previously reported. Histopathology analysis suggested administration with rWISP1 aggravated lung injury characterized by more severe diffuse interstitial edema and more inflammatory cell infiltration (Fig. 4a), increased W/D weight ratio (Fig. 4b) as well as decreased lung EBA permeability (Fig. 4c). Total cell counts (Fig. 4d) and total protein levels (Fig. 4e) were also decreased, indicating severer inflammation compared with control group. Besides, MPO levels were also increased (Fig. 4f). These data indicated administration with rWISP1 aggravated lung injury following hepatic IRI, which contrasts with treatment with anti-WISP1 antibody.

rWISP1 facilitates Proinflammatory cytokine and inhibits anti-inflammatory cytokine production in lung tissue following hepatic IRI

Administration with rWISP1 significantly aggravated lung injury following hepatic IRI, we next wondered 

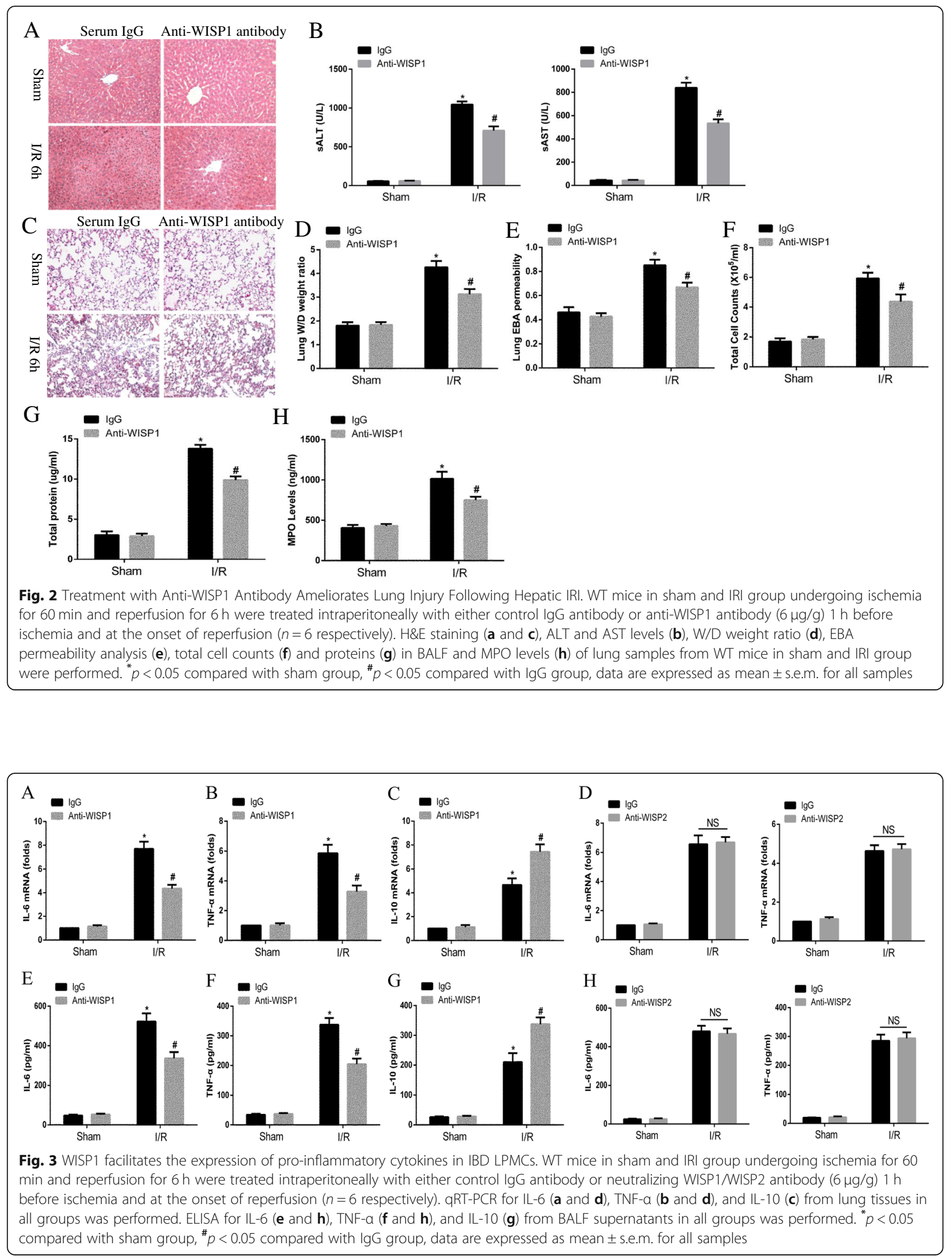


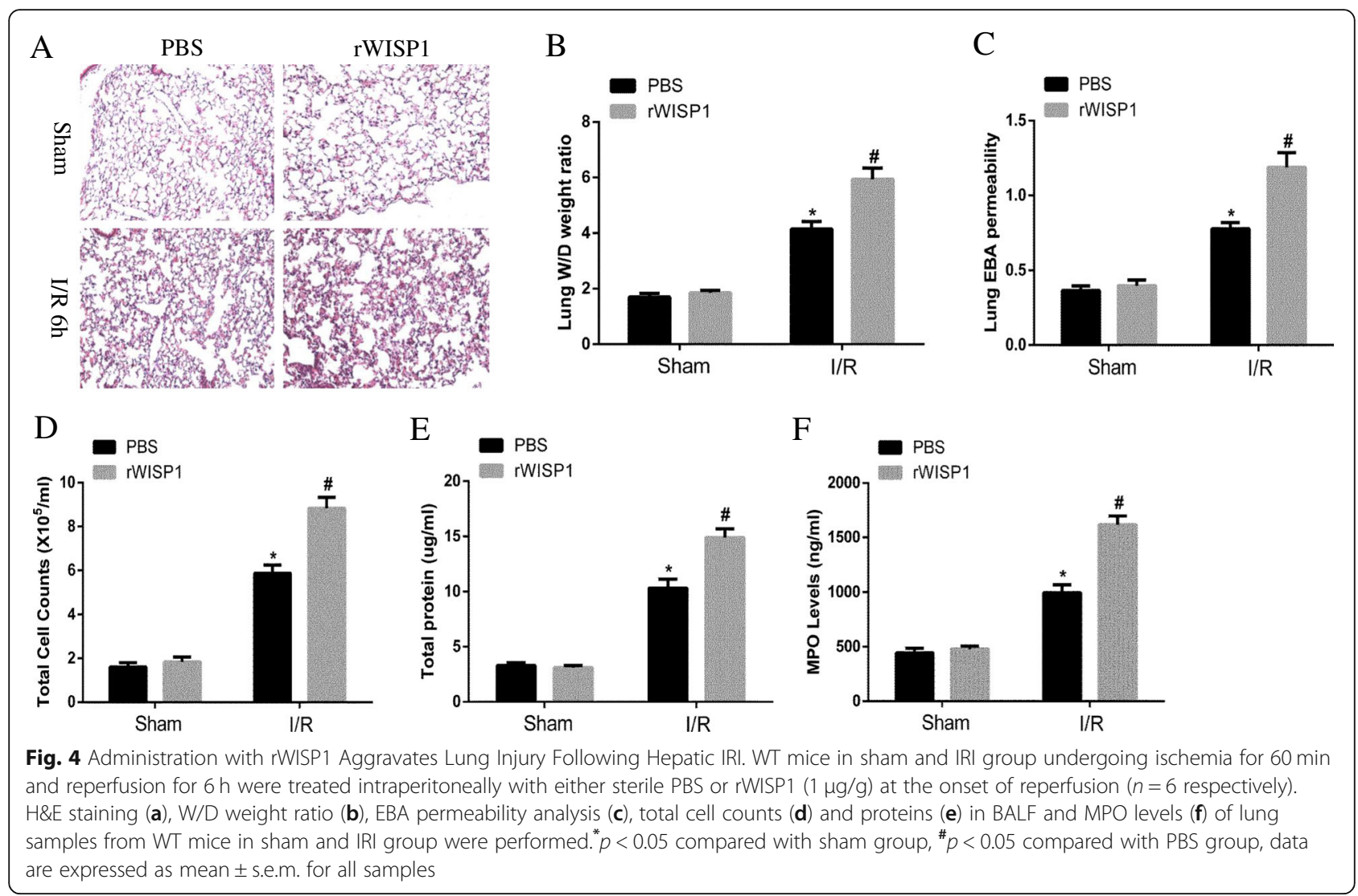

whether rWISP1 could alter inflammatory cytokine production in the lung tissue. Mice were treated as previously described. Lung tissues were harvested, and then qRT-PCR and ELISA analysis were performed. Consistently, qRT-PCR analysis suggested IL-6 (Fig. 5a) and TNF- $\alpha$ (Fig. 5 b) transcript levels were significantly upregulated in lung tissue following hepatic IRI. IL-10 (Fig. 5c) was markedly downregulated. ELISA evaluation in protein level further confirmed the increased levels of IL-6 (Fig. 5d) and TNF- $\alpha$ (Fig. 5e) and decreased levels of IL-10 (Fig. 5f). Collectively, these results demonstrated administration with rWISP1 significantly facilitated proinflammatory cytokine and inhibited anti-inflammatory cytokine production in the lung after hepatic IRI, further validating the pathological role of WISP1.

\section{WISP1 contributes to lung injury in hepatic IRI dependent on TLR4}

Previous work has demonstrated WISP1 ameliorated lung injury in hepatic IRI through increased production of proinflammatory cytokines and inhibition of anti-inflammatory cytokines. We next sought to determine the specific mechanism of WISP1 in regulating inflammatory cascades in the lung. Previous studies have demonstrated that WISP1 interacted with TLR4 and contributed to ventilator-induced lung injury (VILI) dependent on TLR4 signaling [18]. However, the underlying mechanism of WISP1 in mediating lung injury following hepatic IRI still remains unknown. C57BL/6 WT $\left(\mathrm{TLR}^{+/}\right.$in the Fig. 6) and $\mathrm{TLR}^{-1-}$ mice were adopted in the experiment. Both mice were treated with control and IRI operation to determine the expression of WISP1. Interestingly, in IRI (I/R in the Fig. 6) group, WISP1 transcripts (Fig. 6a) and protein expression (Fig. 6a) in $\mathrm{TLR}^{-1-}$ mice was decreased significantly compared with WT mice, indicating WISP1 expression in the lung tissue following hepatic IRI was dependent on TLR4. Next, we would like to examine whether WISP1 mediates inflammatory cascades through TLR4. WT and $\mathrm{TLR}^{-/-}$mice in sham and IRI group were treated with anti-WISP1 antibody and rWISP1 to evaluate lung injury. TLR4 ${ }^{-1-}$ mice displayed decreased W/D ratio (Fig. 6b) and EBA permeability (Fig. 6c) compared with WT mice while no significance was detected between WT and $\mathrm{TLR}^{-/-}$mice in IRI group treated with anti-WISP1 antibody. However, W/D ratio (Fig. 6d) and EBA permeability (Fig. 6e) of TLR4 ${ }^{-/-}$mice in IRI group administered with rWISP1 were significantly decreased compared with those in WT mice, indicating rWISP1 could not induce lung injury in TLR4 ${ }^{-1-}$ mice. Collectively, these date demonstrated WISP1 expression was 

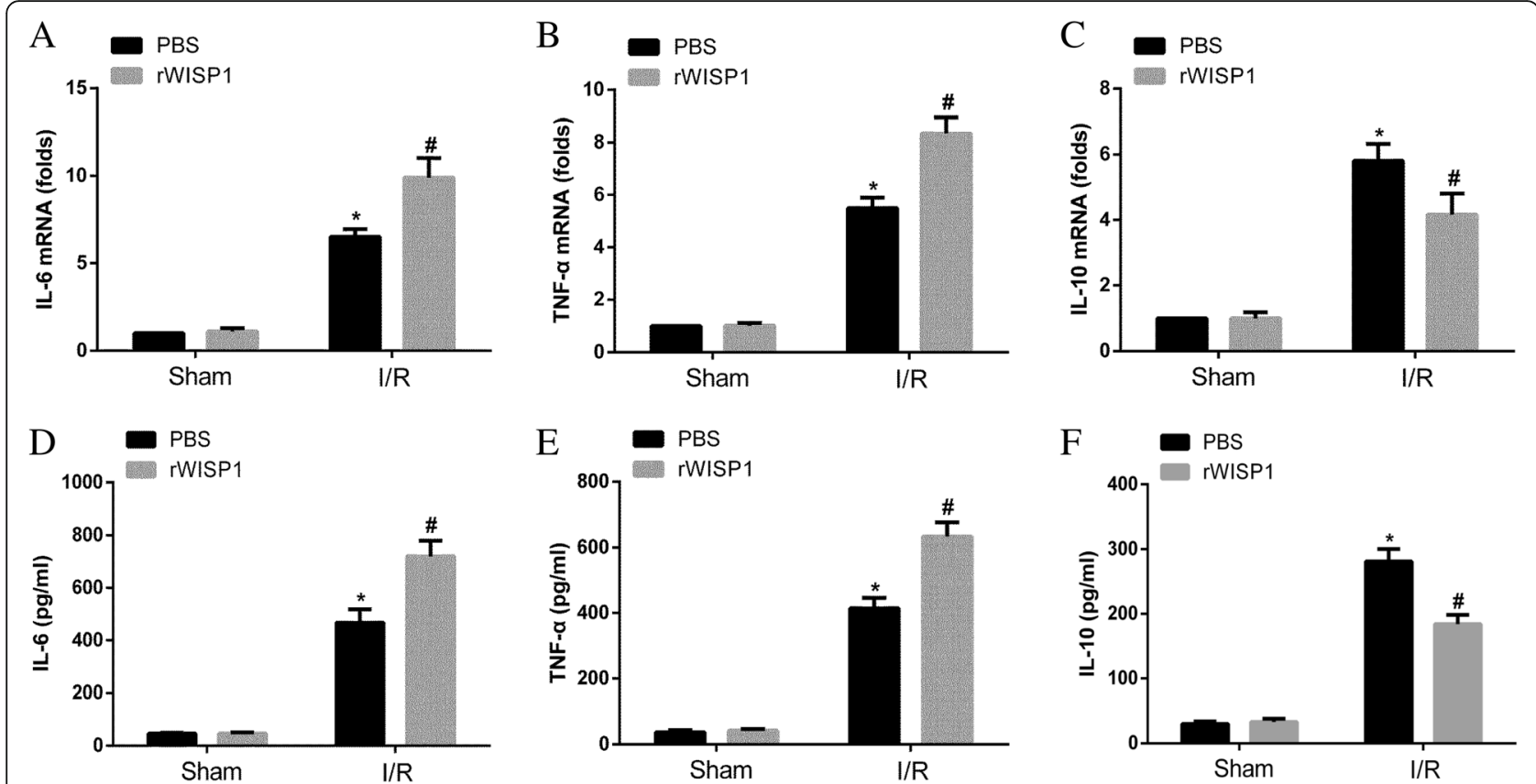

Fig. 5 rWISP1 Facilitates Proinflammatory Cytokine and Inhibits Anti-inflammatory Cytokine Production in Lung Tissue Following Hepatic IRI. WT mice in sham and IRI group undergoing ischemia for $60 \mathrm{~min}$ and reperfusion for $6 \mathrm{~h}$ were treated intraperitoneally with either sterile PBS or rWISP1 $(1 \mu \mathrm{g} / \mathrm{g})$ at the onset of reperfusion ( $n=6$ respectively). qRT-PCR for IL-6 (a), TNF-a (b), and IL-10 (c) from lung tissues in all groups was performed. ELISA for IL-6 (d), TNF-a (e), and IL-10 (f) from BALF supernatants in all groups was performed. $p<0.05$ compared with sham group, ${ }^{\#} p<0.05$ compared with PBS group, data are expressed as mean \pm s.e.m. for all samples

dependent on TLR4 and WISP1 contributed to lung injury in hepatic IRI through TLR4.

\section{Discussion}

Hepatic IRI is a common injury followed by liver transplantation, trauma, shock, etc. Besides liver injury, hepatic IRI also induce remote organ injuries, such as lung injury [8]. The underlying mechanisms of hepatic IRI still remain unclear, of which some mediators have been found playing indispensable roles in liver IRI induced lung injury, including high mobility group box-1 protein (HMGB1), adrenaline and $\mathrm{N}$ acetyl-cysteine [28-30]. In the current study, we reported a new mediator, WISP1, plays a pivotal effect on hepatic IRI induced lung injury.

WNT1 inducible signaling pathway protein 1 (WISP1), one of the most important members in the CCN family, is a secreted extracellular matrix (ECM) which is ubiquitously expressed in various organs and tissues. The molecular function of CCN family has been associated with wound healing, organ fibrosis, cell survival and proliferation [31, 32]. WISP1 has been identified to be involved in the onset of several malignant diseases, such as breast cancer, hepatocellular carcinoma, colon adenocarcinomas, and lung carcinoma, osteoarthritis and lung fibrosis [16, 17, 33, 34]. In addition, we have demonstrated that WISP1 interacts with TLR4 by co-immunoprecipitation and mediates ventilator-induced lung injury (VILI) dependent on TLR4 signaling [18]. We also find WISP1 might contribute to hepatic ischemia reperfusion injury in mice and possibly depends on TLR4/TRIF signaling [24]. However, the role of WISP1 in the lung injury after IRI remains unknown.

This study aims to confirm the role of WISP1 in the lung injury following hepatic IRI and explore the possible regulating mechanisms. Interestingly, we found WISP1 transcript and protein expression were highly increased in the lung tissue following reperfusion for 6 $\mathrm{h}, 12 \mathrm{~h}$ and $24 \mathrm{~h}$ compared with sham mice. Immunofluorescence further confirmed WISP1 expression in the cytoplasm of cell in the lung. These results preliminarily indicated that WISP1 not only participated in the liver injury but also was involved in the pathogenesis of lung injury after hepatic IRI. To further validate the role of WISP1 in the lung injury, mice in the sham and IRI group were treated with anti-WISP1 antibody intraperitoneally to neutralize WISP1 in vivo. Fortunately, we found WISP1 neutralization significantly ameliorated lung injury after hepatic IRI compared to control IgG group characterized by less diffuse interstitial edema and inflammatory cell infiltration. Consistently, treatment with anti-WISP1 antibody markedly inhibited TNF- $\alpha$ and IL- 6 production and enhanced IL-10 expression, further confirming the ameliorated lung injury. Conversely, administration with rWISP1 


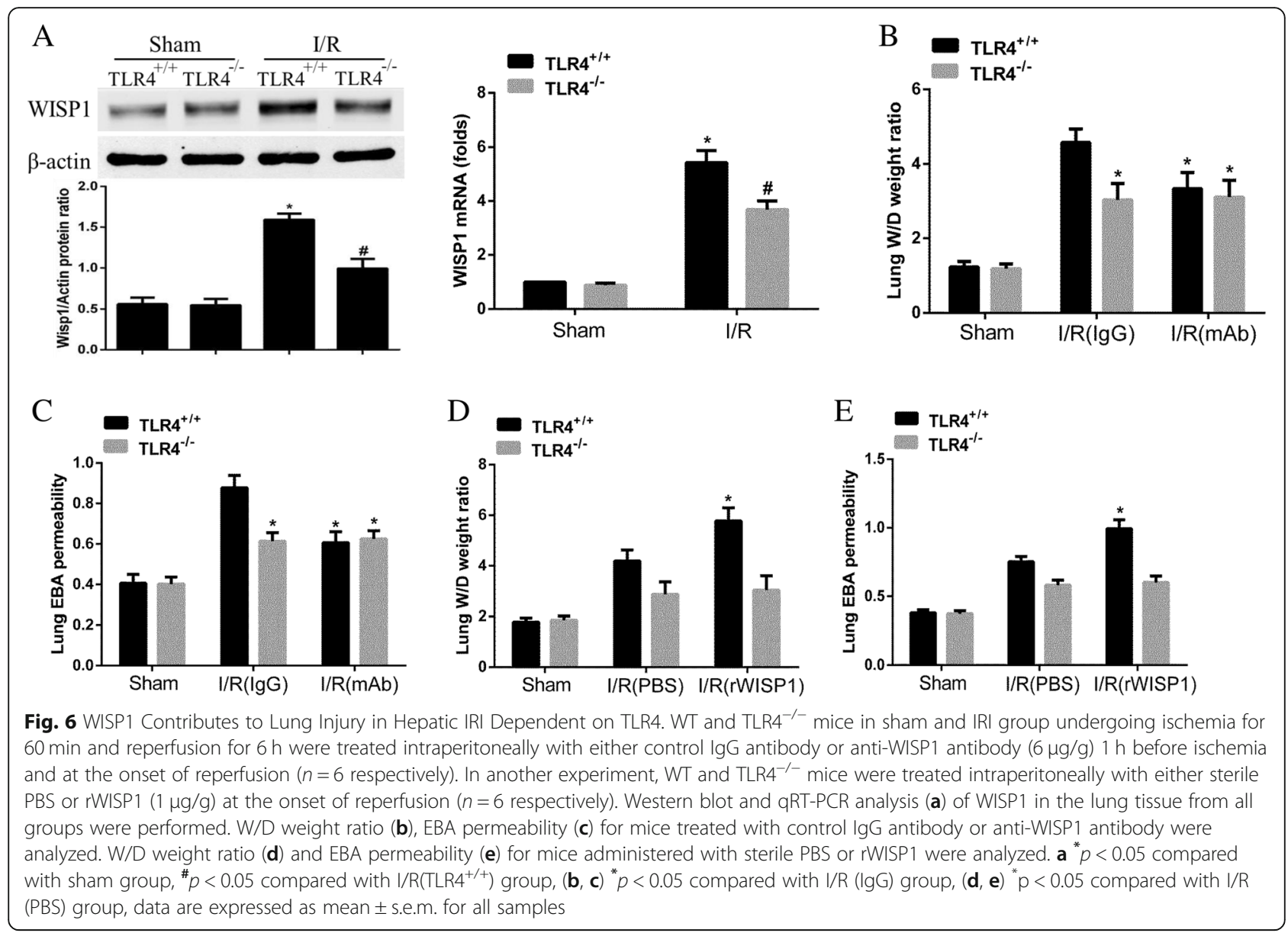

aggravated lung injury together with excessive expression of TNF- $\alpha$ and IL- 6 as well as inhibition of IL-10 production. Since the expression and function of WISP1 has been closely linked to TLR4 pathway, we next sought to explore the potential relationship between WISP1 and TLR4. Interestingly, we found $\mathrm{TLR}^{-/-}$mice in IRI group displayed less transcript and protein expression of WISP1 compared with WT mice, preliminarily suggesting that WISP1 expression in lung injury after hepatic IRI was dependent on TLR4. Besides, rWISP1 could not induce lung injury in $\mathrm{TLR}^{-1-}$ mice of IRI group compared with WT mice, further indicating that WISP1-mediated lung injury and inflammatory cascades might also dependent on TLR4. These data suggested there might be a circulatory regulating mechanism between WISP1 and TLR4 in the lung injury following hepatic IRI. However, the specific mechanisms how TLR4 regulates WISP1 expression and how WISP1 mediates inflammatory cascades through TLR4 need to be further elucidated in future studies.

In conclusion, our study demonstrated that WISP1 expression is upregulated in lung tissue following hepatic
IRI. Anti-WISP1 antibody ameliorated lung injury with alterations in cytokine profiles. rWISP1 aggravated lung injury through excessive proinflammatory cytokine production and inhibition of anti-inflammatory cytokines. WISP1 contributes to lung injury through TLR4 pathway after hepatic IRI. Medications targeting WISP1 might be a promising approach for patients with lung injury following hepatic IRI.

\section{Abbreviations}

BALF: Bronchoalveolar Lavage Fluid; ECM: Extracellular matrix; HMGB1: High mobility group box-1 protein; iNOS: Inducible nitric oxide synthase; IRI: Ischemia-reperfusion injury; MPO: Myeloperoxidase; PRRs: Pattern recognition receptors; ROS: reactive oxygen species; TLR4: Toll-like receptor 4; VILI: Ventilator-induced lung injury; WISP1: WNT1 inducible signaling pathway protein 1

\section{Acknowledgements}

We thank Prof. Timothy R. Billiar (M.D., Ph.D. Professor, Department of Surgery, University of Pittsburgh Medical Center, Pittsburgh, PA) for providing suggestions of this study and supplying us the TLR4 knockout mice.

\section{Funding}

We are very grateful for the support from the National Natural Science Foundation of China (No. 81571927 to Li Q). 


\section{Availability of data and materials}

The dataset of this article are stored in the Laboratory of Shanghai East Hospital and can be made available from the corresponding author upon reasonable request.

\section{Authors' contributions}

QL, YT and ZC designed the study; YT and ZY wrote the manuscript; YT and RZ collected the data; $X D, Z Y$ and $Y T$ performed the experiments and analyzed the data; ZC helped to review the analysis of data; QL and ZC discussed the results and reviewed the manuscript before submission. All authors read and approved the final version of the manuscript.

\section{Ethics approval and consent to participate}

The study was approved by the Ethics Committee of the University of Tongji and the experiments were performed in accordance with the National Institutes of Health Guidelines for the Use of Laboratory Animals.

\section{Consent for publication}

All authors read and approved the final version of the manuscript.

\section{Competing interests}

The authors declare that they have no competing interests.

\section{Publisher's Note}

Springer Nature remains neutral with regard to jurisdictional claims in published maps and institutional affiliations.

Received: 18 December 2017 Accepted: 19 November 2018 Published online: 06 December 2018

\section{References}

1. Bilzer M, Gerbes AL. Preservation injury of the liver: mechanisms and novel therapeutic strategies. J Hepatol. 2000;32:508-15.

2. Caldwell-Kenkel JC, Currin RT, Tanaka Y, Thurman RG, Lemasters J. Reperfusion injury to endothelial cells following cold ischemic storage of rat livers. Hepatology. 1989;10:292-9.

3. Lentsch AB, Kato A, Yoshidome H, McMasters KM, Edwards MJ. Inflammatory mechanisms and therapeutic strategies for warm hepatic ischemia/reperfusion injury. Hepatology. 2000;32:169-73.

4. Colletti LM, Burtch GD, Remick DG, Kunkel SL, Strieter RM, et al. The production of tumor necrosis factor alpha and the development of a pulmonary capillary injury following hepatic ischemia/reperfusion. Transplantation. 1990:49:268-72.

5. Colletti LM, Remick DG, Burtch GD, Kunkel SL, Strieter RM, et al. Role of tumor necrosis factor-alpha in the pathophysiologic alterations after hepatic ischemia/reperfusion injury in the rat. J Clin Invest. 1990;85:1936-43.

6. Chan KC, Lin CJ, Lee PH, Chen CF, Lai YL, et al. Propofol attenuates the decrease of dynamic compliance and water content in the lung by decreasing oxidative radicals released from the reperfused liver. Anesth Analg. 2008:107:1284-9.

7. Chang WJ, Toledo-Pereyra LH. Toll-like receptor signaling in liver ischemia and reperfusion. J Invest Surg. 2012;25:271-7.

8. Kaczorowski DJ, Tsung A, Billiar TR. Innate immune mechanisms in ischemia/reperfusion. Front Biosci. 2009;1:91-8

9. Tsung A, Hoffman RA, Izuishi K, Critchlow ND, Nakao A, et al. Hepatic ischemia/reperfusion injury involves functional TLR4 signaling in nonparenchymal cells. J Immunol. 2005;175:7661-8.

10. Hui W, Jinxiang Z, Heshui W, Zhuoya L, Qichang Z. Bone marrow and nonbone marrow TLR4 regulates hepatic ischemia/reperfusion injury. Biochem Biophys Res Commun. 2009;389:328-32.

11. Bamboat ZM, Balachandran VP, Ocuin LM, Obaid H, Plitas G, et al. Toll-like receptor 9 inhibition confers protection from liver ischemia-reperfusion injury. Hepatology. 2010;51:621-32.

12. Jin $X$, Wang $L$, Wu HS, Zhang L, Wang CY, et al. N-acetylcysteine inhibits activation of toll-like receptor 2 and 4 gene expression in the liver and lung after partial hepatic ischemia-reperfusion injury in mice. Hepatobiliary Pancreat Dis Int. 2007;6:284-9.

13. Zhang JX, Wu HS, Wang H, Zhang JH, Wang Y, et al. Protection against hepatic ischemia/reperfusion injury via downregulation of toll-like receptor 2 expression by inhibition of Kupffer cell function. World J Gastroenterol. 2005;11:4423-6.
14. Pennica D, Swanson TA, Welsh JW, Roy MA, Lawrence DA, et al. WISP genes are members of the connective tissue growth factor family that are upregulated in Wnt-1-transformed cells and aberrantly expressed in human colon tumors. Proc Natl Acad Sci U S A. 1998;95:14717-22.

15. Berschneider $B$, Konigshoff M. WNT1 inducible signaling pathway protein 1 (WISP1): a novel mediator linking development and disease. Int J Biochem Cell Biol. 2011;43:306-9.

16. Margalit $\mathrm{O}$, Eisenbach L, Amariglio N, Kaminski N, Harmelin A, et al. Overexpression of a set of genes, including WISP-1, common to pulmonary metastases of both mouse D122 Lewis lung carcinoma and B16-F10.9 melanoma cell lines. Brit J Cancer. 2003:89:314-9.

17. Calvisi DF, Conner EA, Ladu S, Lemmer ER, Factor VM, et al. Activation of the canonical Wnt/beta-catenin pathway confers growth advantages in c-Myc/ E2F1 transgenic mouse model of liver cancer. J Hepatol. 2005;42:842-9.

18. Li HH, Li Q, Liu P, Liu Y, Li J, et al. WNT1-inducible signaling pathway protein 1 contributes to ventilator-induced lung injury. Am J Respir Cell Mol Biol. 2012;47:528-35.

19. Murahovschi V, Pivovarova O, Illkavets I, Dmitrieva RM, Docke S, et al. WISP1 is a novel adipokine linked to inflammation in obesity. Diabetes. 2015;64:856-66.

20. Nace GW, Huang H, Klune JR, Eid RE, Rosborough BR, et al. Cellular-specific role of toll-like receptor 4 in hepatic ischemia-reperfusion injury in mice. Hepatology. 2013:58:374-87.

21. Ding $X$, Wang $X$, Zhao $X$, Jin $S$, Tong $Y$, et al. RGD peptides protects against acute lung injury in septic mice through Wisp1-integrin beta6 pathway inhibition. Shock. 2015;43:352-60.

22. Zhu GF, Sun B, Niu SF, Cai YY, Lin K, et al. Combined surfactant therapy and inhaled nitric oxide in rabbits with oleic acid-induced acute respiratory distress syndrome. Am J Respir Crit Care Med. 1998;158:437-43.

23. Li H, Su X, Yan X, Wasserloos K, Chao W, et al. Toll-like receptor 4-myeloid differentiation factor 88 signaling contributes to ventilator-induced lung injury in mice. Anesthesiology. 2010;113:619-29.

24. Tong $Y$, Ding $X B$, Chen $Z X$, Jin SQ, Zhao $X$, et al. WISP1 mediates hepatic warm ischemia reperfusion injury via TLR4 signaling in mice. Sci Rep. 2016:6:20141.

25. de Jong PR, Takahashi N, Harris AR, Lee J, Bertin S, et al. Ion channel TRPV1dependent activation of PTP1B suppresses EGFR-associated intestinal tumorigenesis. J Clin Invest. 2014;124:3793-806.

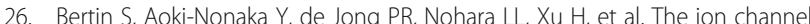
TRPV1 regulates the activation and proinflammatory properties of CD4(+) T cells. Nat Immunol. 2014;15:1055-63.

27. Klebanoff SJ. Myeloperoxidase: friend and foe. J Leukoc Biol. 2005;77:598-625.

28. Yang Z, Deng Y, Su D, Tian J, Gao Y, et al. TLR4 as receptor for HMGB1mediated acute lung injury after liver ischemia/reperfusion injury. Lab Investig. 2013;93(7):792-800,

29. Castro AP, Castro Junior MA, Lauz S, Facin E, Simões Mde J, et al. The role of $\mathrm{N}$-acetyl-cysteine in the lung remote injury after hepatic ischemia and reperfusion in rabbits. Acta Cir Bras. 2012;27(1):49-55.

30. Ota S, Yazawa T, Tojo K, Baba Y, Uchiyama M, et al. Adrenaline aggravates lung injury caused by liver ischemia-reperfusion and high-tidal-volume ventilation in rats. J Intensive Care. 2016;4:8.

31. Chen CC, Lau LF. Functions and mechanisms of action of CCN matricellular proteins. Int J Biochem Cell B. 2009;41:771-83.

32. Jun JI, Lau LF. The matricellular protein CCN1 induces fibroblast senescence and restricts fibrosis in cutaneous wound healing. Nat Cell Biol. 2010;12:676-85.

33. Blom AB, Brockbank SM, van Lent PL, van Beuningen HM, Geurts J, et al. Involvement of the Wnt signaling pathway in experimental and human osteoarthritis prominent role of Wnt-induced signaling protein 1. Arthritis Rheum. 2009:60:501-12.

34. Konigshoff M, Kramer M, Balsara N, Wilhelm J, Amarie OV, et al. WNT1inducible signaling protein-1 mediates pulmonary fibrosis in mice and is upregulated in humans with idiopathic pulmonary fibrosis. J Clin Invest. 2009:119:772-87. 